\title{
Outcome of Salvage Lumbar Fusion after Lumbar Arthroplasty
}

\author{
Hussein Alahmadi, Harel Deutsch \\ Department of Neurological Surgery, Rush University Medical Center, Chicago, IL, USA
}

\begin{abstract}
Study Design: Retrospective review.
Purpose: This study aims to define the role of lumbar fusion for persistent back pains after the lumbar disc replacement.

Overview of Literature: Little is written about lumbar fusion after optimally placed lumbar arthroplasty in patients with persistent lower back pains.

Methods: Retrospective review of cases of lumbar artificial disc requiring subsequent fusion because of persistent back pains despite optimally placed artificial discs. Outcomes were evaluated using Oswestry Disability Index (ODI) and visual analogue scale (VAS). Clinical improvements indicated $25 \%$ improvement in ODI and VAS values.

Results: Five patients met the study criteria. The mean baseline ODI for the five patients was 52 . The mean baseline VAS scores for back and leg pains were 76 and 26, respectively. All the five patients had optimally placed prosthesis. The indication for surgery was the constant low back pains found in all the patients. Revision surgery involved disc explantation and fusion in two of the patients and posterolateral fusion without removing the prosthesis in three. None of the patients achieved adequate pain control after the revision surgery despite the solid bony fusion documented by postoperative computed tomography. The mean ODI value after the fusion was 55. The mean values for back and leg pains VAS were 72 and 30 , respectively.

Conclusions: Lack of good pain relief after successful lumbar artifical disc replacements may indicate different etiology for the back pains. The spine-treating surgeons should have a high threshold level to perform salvage fusion at that level.
\end{abstract}

Keywords: Lumbar vertebrae; Intervertebral disc degeneration; Total disc replacement; Spinal fusion

\section{Introduction}

Lumbar disc replacement (LDR) has been established as a treatment option for patients with discogenic back pain secondary to degenerative disc disease [1-5]. As seen with any technology, LDR is prone to complications or failures that need revision surgery. The vast majority of the literature about revision of LDR involves removal of the artificial disc [6-9]. Most revisions are performed for cases where there is prosthesis migration or disloca- tion. In such cases, the need for artificial disc removal is prominent. While, on the other hand, there are patients who have consistent lower back pains after LDR despite the optimal position of the prosthesis on postoperative imaging. The indications for revision surgery and prosthesis removal in this group have not been defined. Furthermore, little has been written about posterior-alone revisions of LDR without removing the prosthesis. In this report, we reviewed the series of revision surgery for patients with lumbar artificial discs who failed to achieve

Received Oct 8, 2012; Revised Jan 10, 2013; Accepted Jan 19, 2013

Corresponding author: Hussein Alahmadi

Department of Neurological Surgery, Rush University Medical Center,

1725 W. Harrison St., Suite 1115, Chicago, IL 60612, USA

Tel: +1-312-942-6644, Fax: +1-312-942-2176, E-mail: Hussein_alahmadi@yahoo.com 
Table 1. Summary of the management of the five cases

\begin{tabular}{lcccc} 
Case No. & Age (yr)/gender & Level & Revision surgery & Comments \\
\hline 1 & $33 / F$ & $L 4-5$ & L4-S1 PLF & - \\
2 & $38 / F$ & $L 5-S 1$ & L5-S1 PLF & - \\
3 & $41 / F$ & $L 5-S 1$ PLF+ALIF & Some improvement after arthroplasty \\
4 & $40 / F$ & $L 5-S 1$ & $L 5-S 1$ PLF+ALIF & - \\
5 & $55 / F$ & $L 4-5$ & $L 4-5$ PLF & - \\
\hline
\end{tabular}

PLF, posterolateral fusion; ALIF, anterior lumbar interbody fusion.

satisfactory lower back pain control despite the optimal appearance of prosthesis visible on the imaging.

\section{Materials and Methods}

Retrospective review was conducted for our prospectively maintained database for patients who had lumbar artificial disc. We reviewed all patients who had revision surgery after artificial lumbar disc insertions. We reviewed the indication for surgery, clinical and radiological features at initial presentation and at each follow-up. For patients who have had the revision surgeries at other institutions, external records were retrieved accordingly. All patients were scheduled to have regular follow-up visits at 3, 6, 12, and 24 months respectively. Oswestry Disability Index (ODI) and visual analogue score (VAS) for leg and back pains were recorded for each patient at baseline and after each visit. Improvement was defined as at least 25\% improvements in ODI or pain VAS. We considered the artificial disc placement optimum if lumbar spine X-ray shows the prosthesis has a central position within the confines of the disc space with preserved movements on dynamic imaging. There has to be no evidence of endplate fracture, prosthesis subsidence, osteolysis or lucency around the implant.

\section{Lumbar arthroplasty indications}

Our standard indication for lumbar artificial disc is a patient with intractable mechanical lower back pains who failed at least 6 months of non-surgical management. Lower back pain has to be the predominant symptom. The pain should be worse with flexion and prolonged sitting, and relieved by lying down (suggesting that loading the disc space precipitates the pain). The magnetic resonance imaging (MRI) imaging must demonstrate only a single affected level with decreased T2 weighted signal, loss of disc height, and endplate changes. There should be no evidence of advanced facet arthropathy and the dynamic X-ray has to show preserved motion at the index level without segmented instability. If the clinical and radiological pictures pointed to a single level, we did not perform discogram on a routine basis.

\section{Results}

In the past 5 years, 40 patients underwent LDR at our institution for refractory discogenic back pains. Five patients who had LDR at our institution required revision at the operated level due to failure of pain relief. None of the patients who had LDR at our institution required revision surgery for poor prosthesis position or a local complication. All five patients were female. Mean age was 41 years (range, 33-55 years). The main symptom in the five patients was mechanical lower back pains. All patients went through an initial trial of non-surgical pain management before considered revision surgery. The mean baseline ODI before LDR for the five patients was 52 (range, 40-76; stamdard deviation [SD], 17). The mean baseline VAS score was significantly higher for the back as compared to the legs, 76 and 26 , respectively ( $p=0.003$; confidence interval, 21.38-77.02). All patients had MRI scans which showed one level to have advanced degenerative disease out of proportion to the rest of the lumbar spine. One patient had a preoperative discogram that was postive at the most degenerated level on the MRI (case no. 2). The LDR was done for L5-S1 in three cases and L4-5 in the other two cases (Table 1). Three patients had Activ-L artifical disc (Aesculap Inc., Tuttlingen, Germany) and two patients had Charite artificial disc (DePuy Orthopaedics Inc., Raynham, MA, USA). All five patients had the lumbar artificial discs inserted by the same surgeon. None of the cases had prosthesis migration or a local complication secondary to the prosthesis. According 
Table 2. Summary of the ODI and back pain VAS values for the five patients

\begin{tabular}{lcccccc} 
Case No. & $\begin{array}{c}\text { Baseline } \\
\text { ODI }\end{array}$ & $\begin{array}{c}\text { Baseline back } \\
\text { pain VAS }\end{array}$ & $\begin{array}{c}\text { ODI } \\
\text { after LDR }\end{array}$ & $\begin{array}{c}\text { Back pain VAS } \\
\text { after LDR }\end{array}$ & $\begin{array}{c}\text { ODI } \\
\text { after fusion }\end{array}$ & $\begin{array}{c}\text { Back pain VAS } \\
\text { after fusion }\end{array}$ \\
\hline 1 & 40 & 95 & 42 & 83 & 46 & 90 \\
\hline 2 & 76 & 68 & 84 & 94 & 78 & 100 \\
3 & 42 & 88 & 24 & 18 & 44 & 85 \\
4 & 54 & 50 & 42 & 51 & 46 & 38 \\
\hline 5 & 50 & 80 & 45 & 75 & 63 & 50 \\
\hline
\end{tabular}

ODI, Oswestry Disability Index; VAS, visual analogue scale; LDR, lumbar disc replacement.

to four patients, there was no significant improvements in pain control. One of the five patients had significant improvement in ODI and pain VAS (case no. 3). Despite admitting the significant improvement, the patient was not happy with the degree of the residual pain and decided to have the spinal fusion performed at another institution. The mean ODI value for the five patients after LDR was 41 (range, 16-72; SD, 21). The mean values for pain VAS for the back and the leg after LDR were 65 and 23, respectively (Table 2). There were no significant differences in the mean ODI, back and leg pain VAS values before and after the LDR. After the LDR, all the five patients were re-imaged due to the lack of improvements. None of the five patients had evidence of prosthesis dislocation or significant subsidence. And, none of the five patients had constiutional symptoms, elevated white blood cell counts or elevated erythrocyte sedemintation rate to suggest any infections. Three patients had posterolateral fusion with pedicle screws without removing the prosthesis. Two patients had the prosthesis removed, and had anterior interbody fusion and posterolateral fusion with pedicle screws. One of the two patients with the prosthesis removal had the revision surgery done at another institution and the other one was our first revised case. Later we modified our practice to consider posterior fusion without prosthesis removal given the vascular risks associated with anterior revision. One patient (case no. 1), who had L4-L5 LDR, was found later to have worsening degenerative disc disease at L5-S1 and she underwent posterolateral fusion from L4 to S1 with transforaminal lumbar interbody fusion at L5-S1. The avergae duration between the LDR and the fusion was 19 months (range, 3-27 months). The patient who had early revision surgery (within 3 months), had it done at another institution since our recommendation was not to consider revision surgery that early. None of the five patients had significant improvements in their back pains after the fusion. The only patient who had initially significant improvements after the disc replacemnt, had poor pain control after the fusion. There was no vascular or visceral complications in the patients who had the artifical discs removed. The mean ODI value for the five patients after the fusion was 55 (range, 44-78; SD, 15). The mean values for back and leg pains VAS were 72 and 30, respectively. Again, there was no significant differences in the mean values for ODI, back and leg pains VAS at baseline and after the fusion. In three patients, the fusion was done at another institutions. The average follow-up for the five patients was 31 months (range, 24-37 months).

\section{Case illustartion}

A 38-year-old female presented with severe mechanical lower back pain that was exacerbated by prolonged sitting. She did not have significant radicular pains. Her MRI and X-ray showed L5-S1 degenerated disc with loss of disc height and endplate changes (Fig. 1). The patient

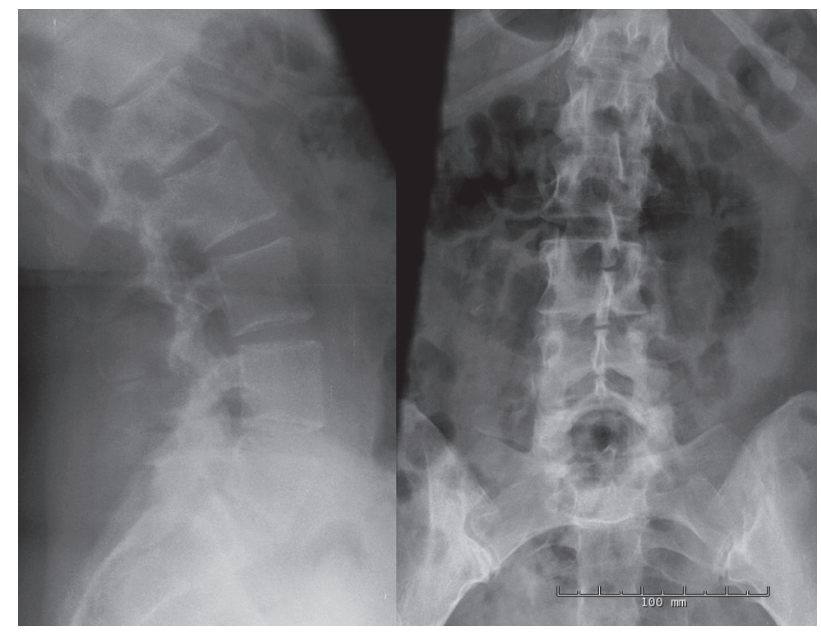

Fig. 1. Preoperative antero-posterior and lateral X-ray showing loss of disc height at L5-S1. 
underwent a diagnostic discogram which provoked pain at L5-S1. The patient failed conservative measures. She underwent L5-S1 LDR with Aesculap disc. After her surgery, the patient's back pain did not improve despite good positioning of the prosthesis on the post-operative imaging (Fig. 2). The patient underwent L5-S1 posterolateral fusion with pedicle screws without removing the artificial disc. The patient had persistent back pains despite the imaging that showed solid bony fusion from L5 to S1 (Fig. 3).

\section{Discussion}

Disc replacement, as any surgical intervension for lumbar degenerative disc disease, is prone to failure and complications. The increase in utilization of this technology among spine surgeons was paralleled by a growth in the experience with revision surgery. Revision surgery for lumbar artifical disc can be associated with risk of vascular or visceral complications because of abdominal adhesions [6]. This could be minimzed by approaching the spine from the contra-lateral side to avoid the adhesions or using an extreme lateral approach $[7,8,10]$. In cases with prosthesis migration or dislocation, the anterior approach for revision surgery and prosthesis explanations are essential. This is usually followed by performing lumbar interbody fusions (with or without posterior fixations) or less likely by inserting another artificial disc [11]. However, when the prosthesis appears in optimal position without complications and the patient still has pain, the indications and the role of revision surgery are less clear. Furthermore, decisions such as surgical approach and the need to remove the prosthesis and perform an interbody fusion lack obvious guidelines.

Our series included patients who failed to achieve adequate pain relief despite optimum positioning of the prosthesis. Identifying the source of pain should be the most important concern before the lumbar arthroplasty. Although the relatively low number of failed cases in our series may reflect our highly selective approach with this technology, a preoperative confirmatory discogram may have helped identifying some of those failures. With the lack of improvements after LDR with optimal position on imaging, it is necessary to identify the source of pain. In all the patients, we ruled out prosthesis-related complications such as subsidence or prosthesis dislocations. In theses cases, pain could be coming from the same level facet joint, other levels, due to psychological factors, or other anatomical substrates. Due to the lack of significant degenerative changes at other levels in our cohort, the pain was attributed to the same level motion and fusion was performed to address facet-related pain (fusion included an adjacent degenerated level found in one of the cases). All five patients achieved solid bony fusions after the revision surgery for LDR but still suffered the universal poor outcome. This indicates that it is unlikely that the pain was from the index level. From such results, we can deduce that the lack of improvement after optimally placed lumbar artificial disc could be a sign that pain is coming from other levels. The spine surgeon should have a relatively higher threshold for fusing the operated level.

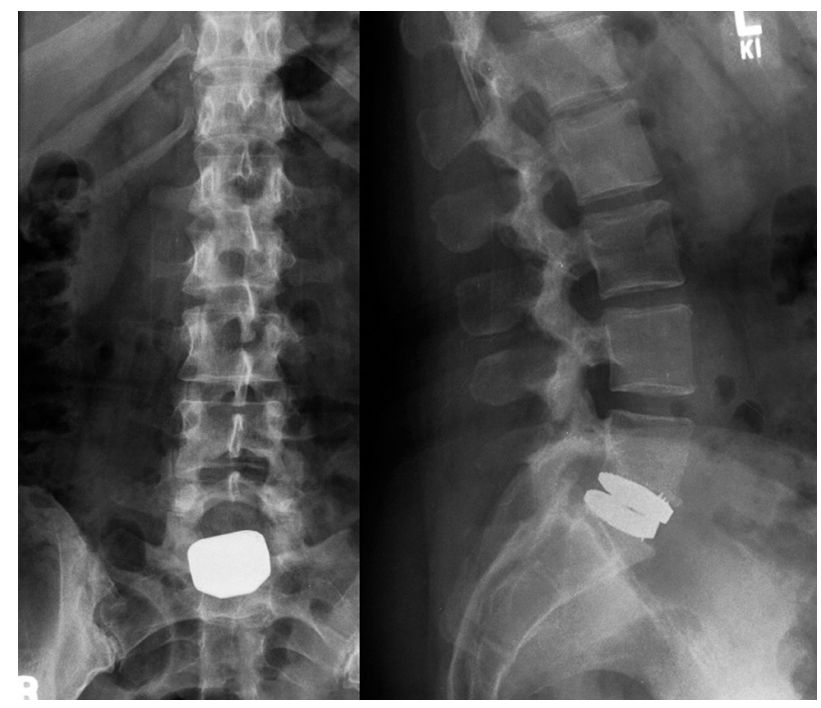

Fig. 2. Lumbar spine antero-posterior and lateral X-ray views showing optimal positioning of the prosthesis.

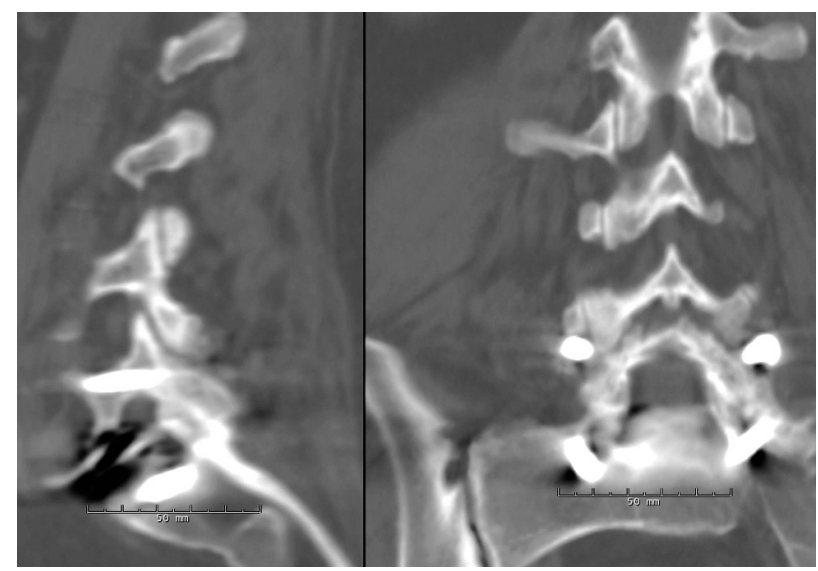

Fig. 3. Lumbar spine computed tomographic scan (parasagittal and coronal views) showing posterolateral bony fusion across the level with artificial disc. 
Rosen et al. [12] reported the results of revision surgery after LDR and suggested that if the facet joint can be confirmed as the cause of pain based on improvement after facet block, the fusion may have the ability to relieve the back pain. We agree that facet block is an important diagnostic tool for those patients and may help predict their outcomes after fusion. Geisler et al. [13] reported a cohort of patients who failed to achieve pain control after technically successesful LDR or anterior lumbar interbody fusion (ALIF) and thus required re-operation. The patients with ALIF were converted to $360^{\circ}$ fusion and patients with LDR received posterior fusion. The patients who required the supplemental fusion continued to have poor pain control as compared to patients who did not require repeated surgery. Similar conclusions with the authors deduce that the lack of improvements after the surgery may suggest different pain etiology from other levels.

In our cohort, three patients had only posterolateral fusion without removal of the artificial discs and eventually developed solid bony fusion. Although the explantation of the artificial disc and performing interbody fusion will likely improve the chance of solid bony fusion, the posterolateral arthrodesis is probably sufficient. By avoiding the removal of prosthesis, the surgeon may be able to spare the patients the added morbidity. For patients where the pain is related to the facet joint, posterolateral fusion alone with pedicle screws should be one of the options for the treating surgeon.

We acknowledge that the retrospective nature of our study and the small number of patients may limit any solid conclusions, and thus larger studies will be necessary to enable us to a better understanding of the role of revised surgery after lumbar arthroplasty.

\section{Conclusions}

Lower back pain is a challenging condition to manage. LDR is one of the treatment options that may fail to achieve pain relief. In cases where lower back pain persists after the LDR, the surgeon should rule out prosthesis-related complications first. If the artifical disc has an optimal placement on postoperative imaging, there should be a high threshold to consider more surgery for the operated level since the lack of improvment may suggest different pain source.

\section{Conflict of Interest}

No potential conflict of interest relevant to this article was reported.

\section{Acknowledgments}

We would like to thank Carol MacPherson, research coordinator at the Department of Neurosurgery at Rush University Medical Center for her help in providing the data.

\section{References}

1. Blumenthal S, McAfee PC, Guyer RD, et al. A prospective, randomized, multicenter Food and Drug Administration investigational device exemptions study of lumbar total disc replacement with the CHARITE artificial disc versus lumbar fusion: part I: evaluation of clinical outcomes. Spine (Phila Pa 1976) 2005;30:1565-75.

2. McAfee PC, Cunningham B, Holsapple G, et al. A prospective, randomized, multicenter Food and Drug Administration investigational device exemption study of lumbar total disc replacement with the CHARITE artificial disc versus lumbar fusion: part II: evaluation of radiographic outcomes and correlation of surgical technique accuracy with clinical outcomes. Spine (Phila Pa 1976) 2005;30:1576-83.

3. Guyer RD, McAfee PC, Banco RJ, et al. Prospective, randomized, multicenter Food and Drug Administration investigational device exemption study of lumbar total disc replacement with the CHARITE artificial disc versus lumbar fusion: five-year followup. Spine J 2009;9:374-86.

4. Zigler J, Delamarter R, Spivak JM, et al. Results of the prospective, randomized, multicenter Food and Drug Administration investigational device exemption study of the ProDisc-L total disc replacement versus circumferential fusion for the treatment of 1-level degenerative disc disease. Spine (Phila Pa 1976) 2007;32:1155-62.

5. Sasso RC, Foulk DM, Hahn M. Prospective, randomized trial of metal-on-metal artificial lumbar disc replacement: initial results for treatment of discogenic pain. Spine (Phila Pa 1976) 2008;33:123-31.

6. Wagner WH, Regan JJ, Leary SP, et al. Access strat- 
egies for revision or explantation of the Charite lumbar artificial disc replacement. J Vasc Surg 2006;44:1266-72.

7. Pimenta L, Diaz RC, Guerrero LG. Charite lumbar artificial disc retrieval: use of a lateral minimally invasive technique. Technical note. J Neurosurg Spine 2006;5:556-61.

8. Leary SP, Regan JJ, Lanman TH, Wagner WH. Revision and explantation strategies involving the CHARITE lumbar artificial disc replacement. Spine (Phila Pa 1976) 2007;32:1001-11.

9. Jeon SH, Choi WG, Lee SH. Anterior revision of a dislocated ProDisc prosthesis at the L4-5 level. J Spinal Disord Tech 2008;21:448-50.

10. Cheng WK, Jadhav V, Palmer DK. A novel modifica- tion for removal of the polyethylene core in artificial disc retrieval using a transpsoas minimally invasive technique. J Neurosurg Spine 2011;14:466-9.

11. David T. Revision of a Charite artificial disc 9.5 years in vivo to a new Charite artificial disc: case report and explant analysis. Eur Spine J 2005;14:507-11.

12. Rosen C, Kiester PD, Lee TQ. Lumbar disk replacement failures: review of 29 patients and rationale for revision. Orthopedics 2009;32. http://dx.doi. org/10.3928/01477447-20090624-08.

13. Geisler FH, Guyer RD, Blumenthal SL, et al. Patient selection for lumbar arthroplasty and arthrodesis: the effect of revision surgery in a controlled, multicenter, randomized study. J Neurosurg Spine 2008;8:13-6. 